\title{
Efficacy of Integrated Intervention Program on Mental Health of Cancer Patients
}

\author{
Bibin Chand ${ }^{1}$, Madhavi Sharma', Narendra S Thagunna ${ }^{2}$ \\ ${ }^{1}$ Institute of Chartered Financial Analysts of India University, Tripura, India, ${ }^{2}$ Psychdesk Foundation, Kathmandu, \\ Nepal
}

\section{Corresponding author:}

Bibin Chand, PhD

Institute of Chartered Financial Analysts of India University, Tripura, India

Email: bibinairforce@gmail.com

\begin{abstract}
Introduction

Copious researchers have made notable findings on the mental health of cancer patients and effect of various discrete therapies, but still there exist a significant paucity on integrated therapeutic interventions over their mental health. The objectives of this study were to find the prevalence of mental health morbidity and the efficacy of an integrated intervention program comprised of Aaron Beck's Cognitive Behavior Therapy (CBT), Jacobson Progressive Muscle Relaxation Therapy (JPMRT) and self- imagery training on the mental health of cancer patients.
\end{abstract}

\section{Methods}

In this cross-sectional study, data was collected from 20 patients diagnosed with cancer aged between 40-55 years from an outpatient department of Cancer Institute, India. General Health Questionnaire by Goldberg \& Hiller, 1979 (GHQ-28) was administrated to assess the mental health in four domains viz: somatic complaints, anxiety and insomnia, social dysfunction and severe depression. Integrated intervention program was conducted for four days per week for one month. Data was analyzed statistically by using normal probability curve (NPC) and paired t-test.

\section{Results}

An overall prevalence of negative mental health and significant positive effect by the integrated intervention on the mental health among cancer patients have been statistically corroborated in this pre-post research.

\section{Conclusion}

Integrated intervention program is efficacious in improving mental health in cancer patients.

Keywords: Cancer, cognitive behaviour therapy, integrated intervention, JPMR, mental health 